\title{
Golang Multithreading
}

\author{
Mykhaylo Bavdys \\ dept. of Electronics and Computer Technologies \\ Ivan Franko National university of Lviv \\ Lviv, Ukraine \\ bavdysmyh@ukr.net
}

\begin{abstract}
In the thesis the issues of development of microservices on the basis of object-oriented design are considered. The peculiarities of creating the layers of logic were described. The methods of research and implementation of the information system for the development of software products for the system on the basis of microservices in the style of object-oriented design have been analyzed, namely: the requirements to the information system; the database structure; the layer structure; architecture of microservice; the structure of the administrative part of the system; the description of the restriction of access to data. The work is based on the Golang language. The coursework contains the following sections: basic concepts of development tools, description of the project, project testing. In the course of the work, conclusions and suggestions were made on improving the development of information systems and the use of microservice architecture development tools in the style of objectoriented design to achieve the set goals.
\end{abstract}

Index Terms - software framework, database, main tool, programming, microservice, architecture, Go, web server, Nginx, analysis, abstract layer.

\section{I.INTRODUCTION}

Currently research on the Go language and the multithreaded Go-language experiments are currently relevant. The main purpose of the study of multi-threaded Go language is carrying information and ensuring the relevance and usefulness of this information.

\section{MultithreADING IN GO}

When initially launched, Golang uses one thread, using its own scheduler and asynchronous calls. (The programmer acquires a sense of multithreading and parallelism.) In this case, the channels run very fast. But if you specify Go to use 2 or more threads then Go starts using lockdowns and channel performance may drop.

This is a big disadvantage. Moreover, most third-party libraries use channels any time it's convenient. Therefore, it is often effective to launch Go with one channel, as it is done by

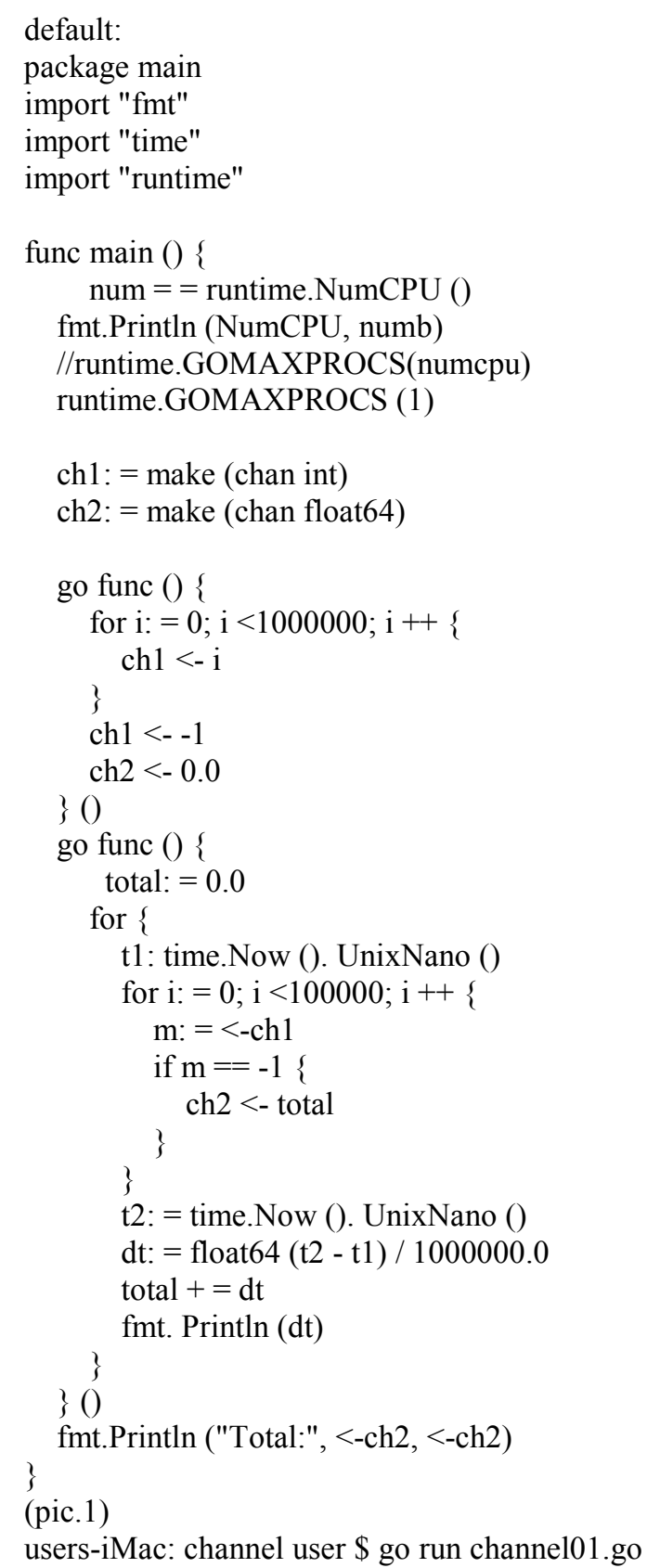




$\begin{array}{ll}\text { NumCPU } 4 & 23.807 \\ 23.901 & 24.039 \\ 24.189 & 23.854 \\ 23.957 & 23.798 \\ 24.072 & 24.1 \\ 24.001 & \text { Total: } 239.7180\end{array}$

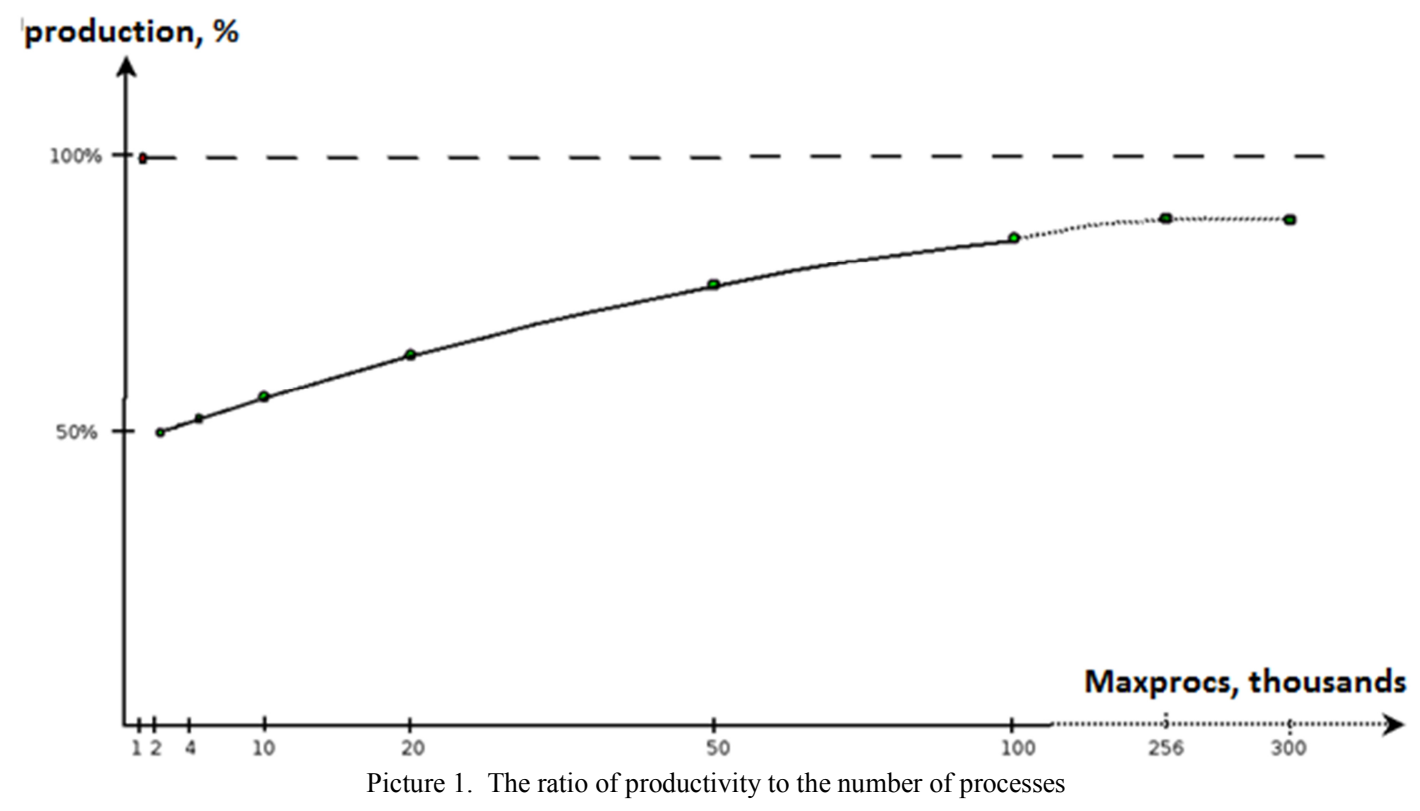

Now, with the active use of all the cores we'll add a 9.178 comment to the following lines:

9.84

9.869

runtime.GOMAXPROCS (numcpu)

9.461

9.802

$\begin{array}{ll}\text { users-iMac: channel user \$ go run channel01.go } & 9.743 \\ \text { NumCPU } 4 & 9.877\end{array}$

543.092

9.756

534.985

Total: 096.848

535.799

533.039

result with 4 threads

538.806

533.315

536.501

533.261

537.73

532.585

rs-iMac: channel user \$ go run channel01.go NumCPU 4

17.046

17.046

16.71

16.315

16.542

16.643

17.69

16.387

17.162

15.232

Let's set it to 100 .

ch1: = make (chan int, 100)

Total: 0 166.77300000000002

users-iMac: channel user \$ go run channel01.go

NumCPU 4

9.704

Example "Thread of threads"

9.618 

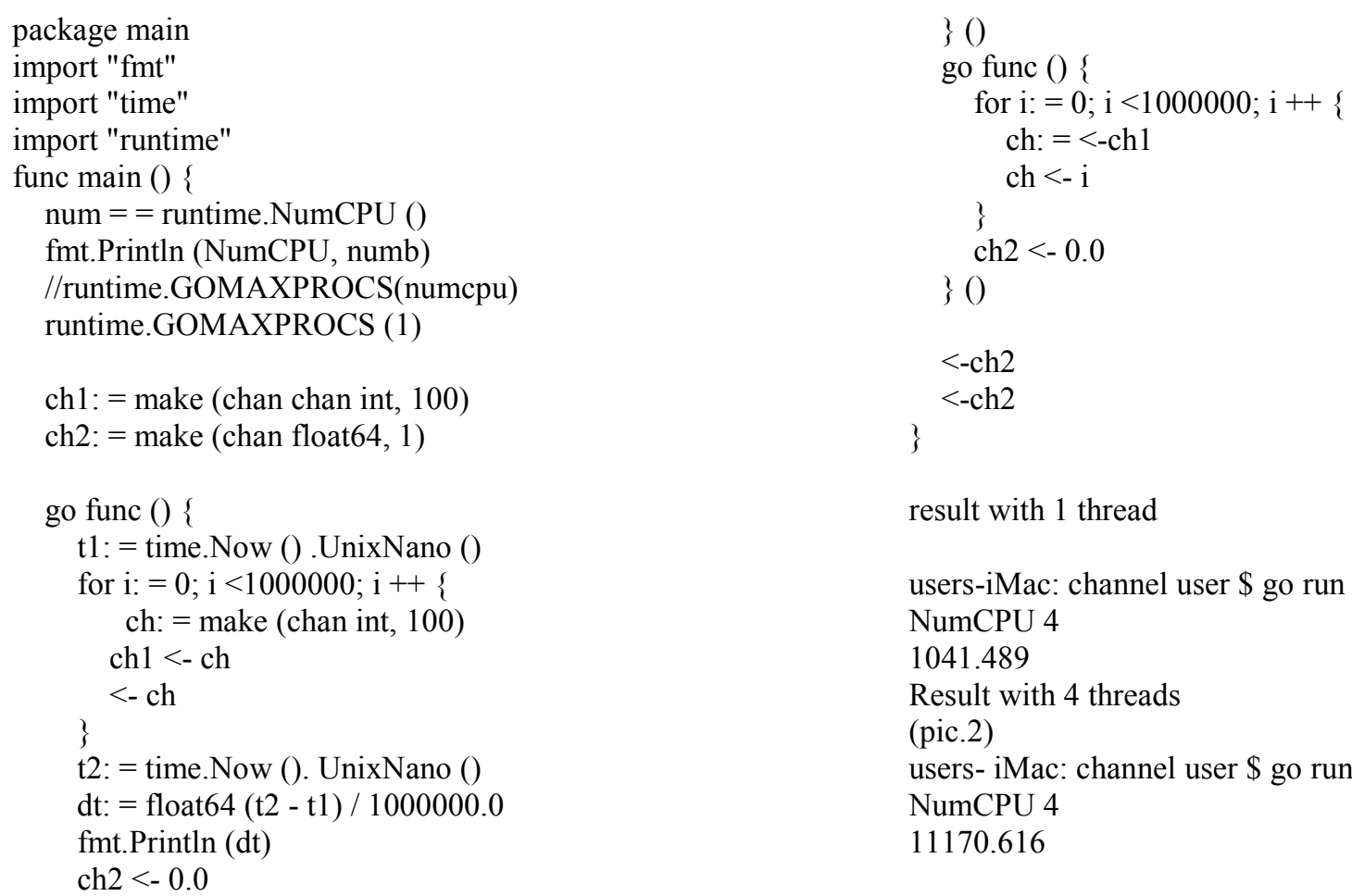

result with 1 thread

users-iMac: channel user $\$$ go run channel03.go NumCPU 4

1041.489

Result with 4 threads

(pic.2)

users- iMac: channel user \$ go run channel03.go

NumCPU 4

11170.616

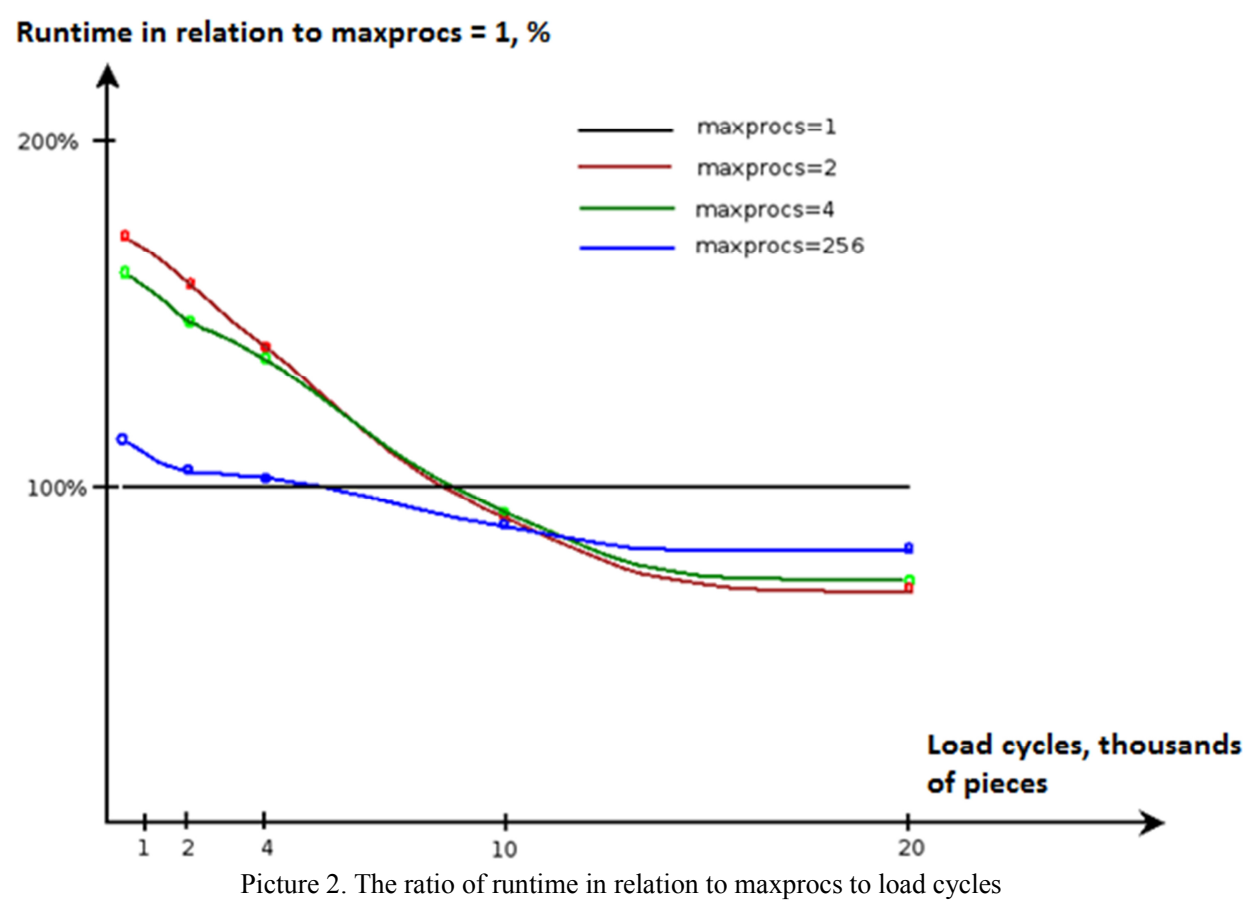

\section{CONCLUSIONS}

So if you have 8 cores and you write a server using Go, you do not have to rely entirely on parallelizing the program. It is better to run 8 single-threaded processes, with a balancer in front of them.

What do these figures mean? The task was to handle 3000 requests per second in the same context (for example, to issue for each query sequentially the following numbers: $1,2,3,4,5 \ldots)$ and the productivity of 3000 requests per second is limited primarily to channels. With the addition of threads and cores, productivity is growing not as quickly as it would be desirable.

\section{REFERENCES}

[1] The Go Programming Language. [Electronical resource]. - Access mode: https://golang.org/doc/

[2] BSD Licenses. [Electronical resource]. - Access mode: $\mathrm{ftp} / / / \mathrm{ftp} . c s . b e r k e l e y . e d u / p u b / 4 b s d / R E A D M E$.Impt.License.Change

[3] Go at Google: Language Design in the Service of Software Engineering. [Electronical resource]. - Access mode: https://talks.golang.org/2012/splash.article 